\title{
Dynamical low rank approximation of time dependent random PDEs
}

Eleonora Musharbash ${ }^{\dagger}$, Fabio Nobile $^{\dagger}$, Eva Vidličková $^{\dagger}$

${ }^{\dagger}$ MATH-CSQI, École Polytechnique Fédérale de Lausanne, Switzerland

\section{Motivation}

Problem setting:

Physical space $D \subset \mathbb{R}^{d}, d=1,2,3$; time interval $\mathcal{T}=[0, T]$; random variables $\xi=\left(\xi_{1}, \ldots, \xi_{N}\right) \in \Gamma$ with a joint density function $\rho \in L^{\infty}(\Gamma)$

$$
\begin{array}{rlrl}
\frac{\partial u(x, t, \xi)}{\partial t} & =\mathcal{L}(u(x, t, \xi), \xi), & x \in D, t & \in \mathcal{T}, \xi \in \Gamma \\
u(x, 0, \xi) & =u_{0}(x, \xi), & x \in D, \xi \in \Gamma \\
u(x, t, \xi) & =h(x, t), & x \in \partial D, t & \in \mathcal{T}, \xi \in \Gamma
\end{array}
$$

\begin{tabular}{|c|c|}
\hline $\begin{array}{l}\text { - POD (spatial basis fixed in time) } \\
\qquad u_{R}(x, t, \xi)=\bar{u}_{R}(x, t)+\sum_{i=1}^{R} Y_{i}(t, \xi) U_{i}(x), \\
\text { where }\left\{U_{i}(x)\right\}_{i=1}^{R} \text { orthogonal functions in } \\
L^{2}(D)\end{array}$ & $\begin{array}{l}\text { - PCE (stochastic basis fixed in time) } \\
\qquad u_{R}(x, t, \xi)=\bar{u}_{R}(x, t)+\sum_{i=1}^{R} Y_{i}(\xi) U_{i}(x, t) \text {, } \\
\text { where }\left\{Y_{i}(\xi)\right\}_{i=1}^{R} \text { zero mean stochastic processes } \\
\text { orthonormal in } L_{\rho}^{2}(\Gamma)\end{array}$ \\
\hline $\begin{array}{l}\text { - Issues } \\
\text { solution manifold changes over time } \rightarrow \mathrm{R} \text { too high }\end{array}$ & $\begin{array}{l}\bullet \text { Issues } \\
\text { "curse of dimensionality", long time integration }\end{array}$ \\
\hline
\end{tabular}

Goal: Low rank approximation

\section{Introduction to DLR}

Goal: Dynamical low rank approximation

$$
u_{R}(x, t, \xi)=\bar{u}_{R}(x, t)+\sum_{i=1}^{R} Y_{i}(t, \xi) U_{i}(x, t),
$$

where $\bar{u}_{R}(x, t)=\mathbb{E}\left[u_{R}(x, t, \xi)\right],\left\{Y_{i}(t, \xi)\right\}_{i=1}^{R}$ are zero mean stochastic processes in $L_{\rho}^{2}(\Gamma)$, $\left\{U_{i}(x, t)\right\}_{i=1}^{R}$ are orthonormal functions in $L^{2}(D)$.

DO condition:

$$
\begin{gathered}
\left\langle\frac{\partial U_{i}(\cdot, t)}{\partial t}, U_{j}(\cdot, t)\right\rangle_{L^{2}(D)}=0, \quad \forall i, j=1, \ldots, R, \forall t \in \mathcal{T} \\
\Longrightarrow \text { unique representation of (1) up to rotation of the basis }\left\{U_{i}\right\}_{i=1}^{R}
\end{gathered}
$$

Dynamically orthogonal approximation:

$$
\begin{aligned}
\frac{\partial \bar{u}_{R}(x, t)}{\partial t} & =\mathbb{E}\left[\mathcal{L}\left(u_{R}(x, t, \xi)\right)\right] \\
\frac{\partial U_{i}(x, t)}{\partial t} & =\mathbb{E}\left[\mathcal{L}\left(u_{R}(x, t, \xi)\right) Y_{i}(t, \xi)\right], \\
\sum_{j=1}^{R} \mathbf{M}_{i j} \frac{\partial Y_{j}(t, \xi)}{\partial t} & =\Pi_{\mathcal{Y}}^{\perp}\left\langle\mathcal{L}^{*}\left(u_{R}(x, t, \xi), \xi\right), U_{i}(x, t)\right\rangle_{L^{2}(D)},
\end{aligned}
$$$$
\forall i=1, \ldots, R
$$$$
\forall i=1, \ldots, R
$$

where $\mathcal{Y}=\operatorname{span}\left\{Y_{1}(t, \xi), \ldots, Y_{R}(t, \xi)\right\}, \Pi \frac{\perp}{\mathcal{Y}}$ is the orthogonal projection operator in $L_{\rho}^{2}(\Gamma)$ to the complement of $\mathcal{Y}, \mathcal{L}^{*}(\cdot)=\mathcal{L}(\cdot)-\mathbb{E}[\mathcal{L}(\cdot)]$ and $\mathbf{M}_{i j}=\left\langle U_{i}(\cdot, t), U_{j}(\cdot, t)\right\rangle_{L^{2}(D)}$

\section{Geometrical interpretation}

- The set of all zero mean $R$-rank random fields form a differential manifold:

$$
\mathcal{M}_{R}=\left\{u_{R}^{*}=\sum_{i=1}^{R} U_{i} Y_{i}, \quad U_{i}, Y_{i} \text { as in (1) }\right\} .
$$

- $u_{R}$ is the DO solution $\Longleftrightarrow$
$\frac{\partial u_{R}(x, t, \xi)}{\partial t}=\mathbb{E}\left[\mathcal{L}\left(u_{R}(x, t, \cdot)\right)\right]+P_{u_{R}^{*}(t)}\left(\mathcal{L}^{*}\left(u_{R}(x, t, \xi)\right)\right)$

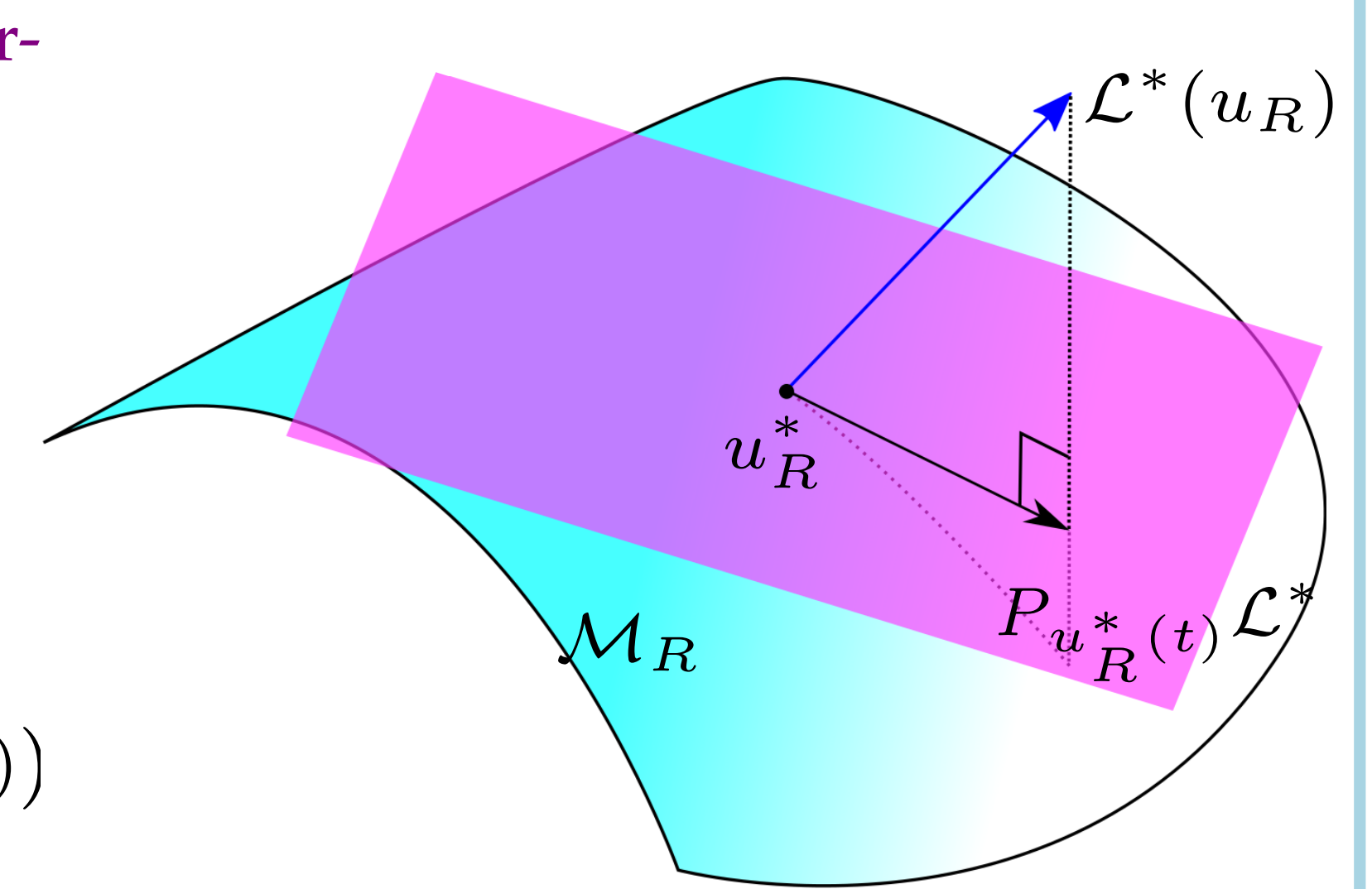

where $P_{u_{R}^{*}(t)}$ is the orthogonal projection onto the tangent space of $\mathcal{M}_{R}$ at $u_{R}^{*}=u_{R}-\mathbb{E}\left[u_{R}\right][1,3]$

\section{Discretization}

- FEM for spatial discretization, semi-implicit Euler for time discretization of $\bar{u}_{R}(\cdot, t), U_{i}(\cdot, t)$ and ex plicit Euler for $Y_{i}(\cdot, t)$

- sparse grid for stochastic discretization

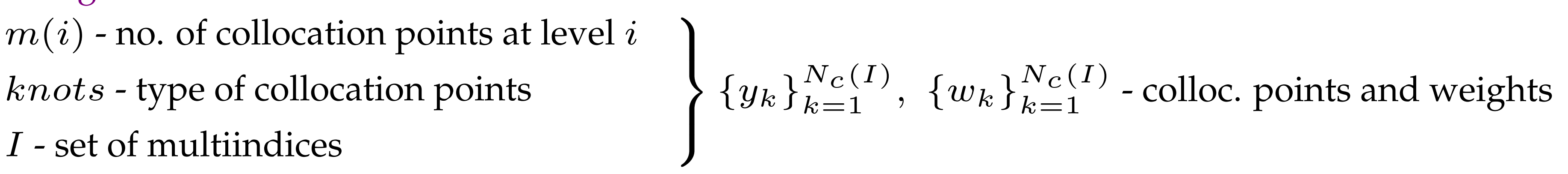

- sparse grid interpolant $S_{I}: L_{\rho}^{2}(\Gamma) \cap C^{0}(\Gamma) \rightarrow \bigoplus_{i \in I} \mathbb{P}_{m(i)-1}(\Gamma)$

$$
\begin{aligned}
S_{I}[f](\xi) & =\sum_{i \in I} \triangle^{m(i)}[f](\xi)=\sum_{k=1}^{N_{c}(I)} f\left(y_{k}\right) L_{k}(\xi) \\
\triangle^{m(i)} & =\bigotimes_{n=1}^{N}\left(\mathcal{U}_{n}^{m\left(i_{n}\right)}-\mathcal{U}_{n}^{m\left(i_{n}-1\right)}\right),
\end{aligned}
$$

where $\left\{L_{k}(\xi)\right\}_{k=1}^{N_{c}(I)}$ - Lagrange polynomials, $\mathcal{U}_{n}^{l}$ - polynomial interpolant of order $l-1$ in the n-th variable $\xi_{n}$.

- sparse grid quadrature: $\mathbb{E}\left[S_{I}[f]\right]=\sum_{k=1}^{N_{c}(I)} f\left(y_{k}\right) w_{k}$

\section{Adaptive choice of sparse grid}
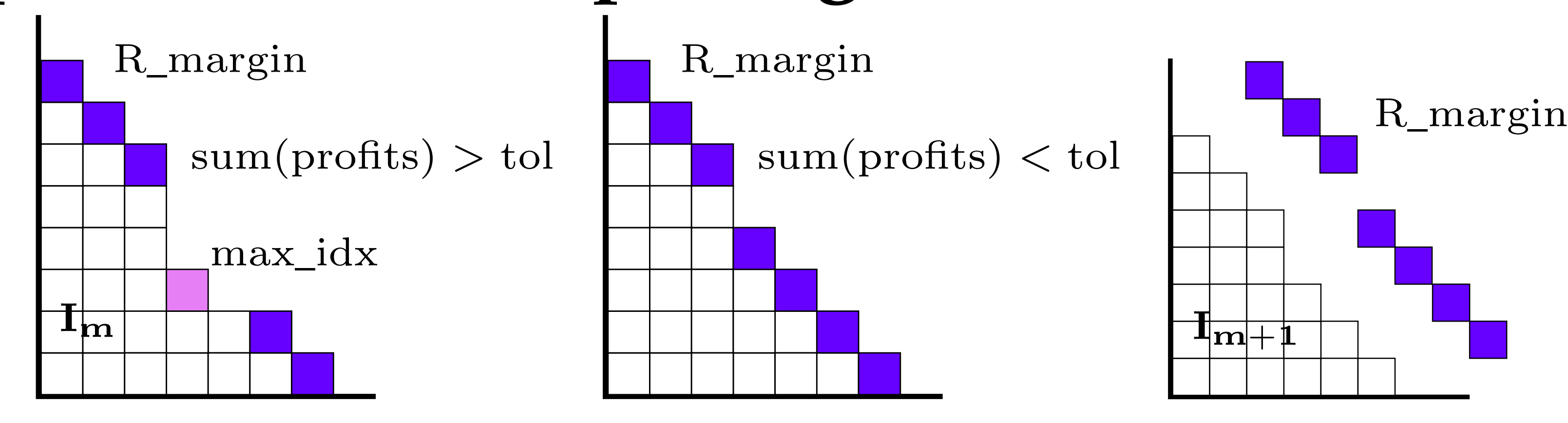

- time discretization $0=t_{0}<t_{1}<\cdots<t_{M}=T$

Goal: multiindex set $I_{m+1}$ for sparse grid $S_{I_{m+1}}$ at time $t_{m+1}$

Data: multiindex set $I_{m}$ from previous time step; so far algorithm works only with $I_{m} \subseteq I_{m+1}$, nested collocation points

Motivation: adapt w.r.t function $\mathbf{f}$

choose $I \supset I_{m}$ s.t.

$$
f(\xi)=\sum_{i \in \mathbb{N}^{N}} \triangle^{m(i)}[f](\xi),
$$

$$
\left\|f-S_{I}[f]\right\| \leq \sum_{i \in \mathbb{N}_{+}^{N} \backslash I}\left\|\triangle^{m(i)}[f]\right\| \approx \sum_{i \in \mathrm{R} \_\operatorname{margin}(I)}\left\|\triangle^{m(i)}[f]\right\|<\text { tol },
$$

where R_margin $(I)=\left\{i \in \mathbb{N}_{+}^{N} \backslash I: i-e_{n} \in I\right.$, for all $n=1, \ldots, N$ with $\left.i_{n}>1\right\}$ [2]

Profit function: profit_function $(I)=\left\{\left\|\triangle^{m(i)}[f]\right\|\right\}_{i \in I}$

Adapted_sparsegrid $\left(\mathrm{I}_{\mathrm{m}}, \mathrm{R} \_\right.$margin, profit_function, tol $)$

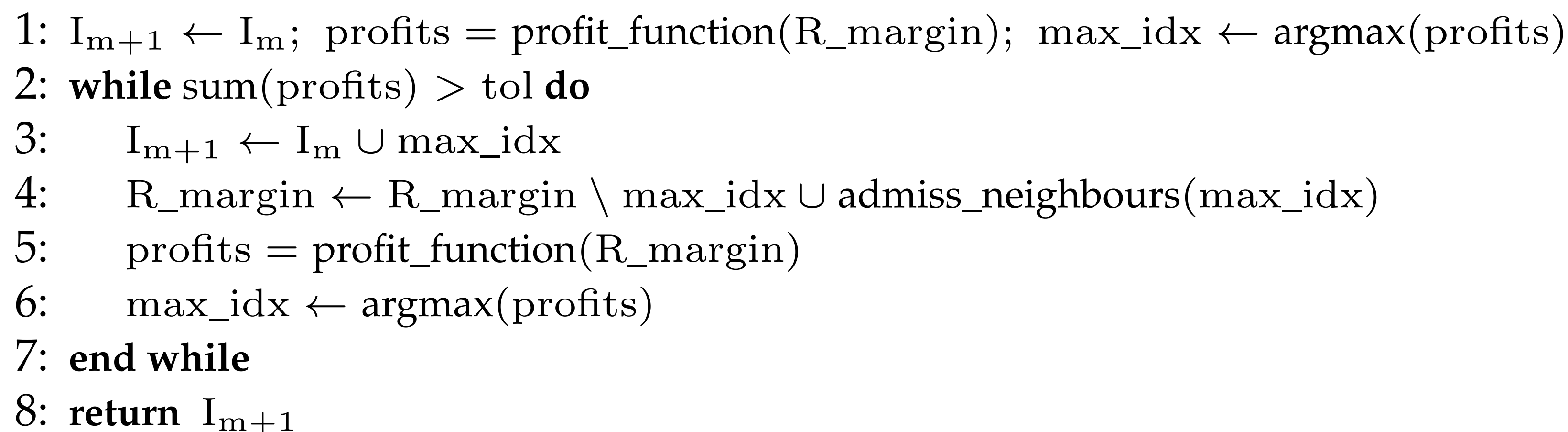

\section{Sparse grid adaptivity within DLR}

Function $\mathbf{f}$ - unprojected right hand side of $(4)$ :

$$
\begin{aligned}
& \text { Pro } \\
& \text { To } \\
& \text { To }
\end{aligned}
$$

$$
\begin{aligned}
f(\xi) & =\left(f_{1}(\xi), \ldots, f_{R}(\xi)\right)^{T} \\
f_{i}(\xi) & =\left\langle\mathcal{L}\left(u_{R}(x, t, \xi), \xi\right), U_{i}(x, t)\right\rangle_{L^{2}(D)}
\end{aligned}
$$$$
\text { profit_function }{ }^{2}(i)=\left\|\triangle^{m(i)}[f]\right\|_{L_{\rho}^{2}}^{2} \approx \sum_{k=N_{c}\left(I_{m}\right)}^{N_{c}\left(I_{m} \cup i\right)}\left\|f\left(y_{k}\right)-S_{I_{m}}[f]\left(y_{k}\right)\right\|_{l^{2}\left(\mathbb{R}_{R}\right)}^{2} \rho\left(y_{k}\right)
$$

Numerical difficulties

To compute accurately the projections $\Pi_{\mathcal{Y}}^{\perp}\left\langle\mathcal{L}, U_{i}\right\rangle_{L^{2}(D)}$ in (4) and the right hand side in (3) one needs a good enough approximation of $\mathbb{E}\left[Y_{j} Y_{k}\right]$. However, unless Gaussian points are used:

$$
\left\|\mathbb{E}\left[Y_{j} Y_{k}\right]-\mathbb{E}\left[S_{I}\left[Y_{j} Y_{k}\right]\right]\right\|_{F r o b} \gg 0 .
$$

Solution: modal representation:

$$
Y_{j}(\xi)=\sum_{l=1}^{K} a_{j}^{l} \psi_{l}(\xi), \quad \psi_{l} \text {-ON polynomials in } \quad L_{\rho}^{2}(\Gamma)
$$

then

$$
\mathbb{E}\left[Y_{j} Y_{k}\right]=\sum_{l=1}^{K} a_{j}^{l} a_{k}^{l} \quad \rightarrow \quad \text { compute } a_{j}^{l} \quad \forall j=1, \ldots, R ; l=1, \ldots, K .
$$

\section{Numerical results

$$
\begin{aligned}
\frac{\partial u(x, t, \xi)}{\partial t} & =\nabla \cdot(a(x, \xi) \nabla u(x, t, \xi)), & x \in[0,1]^{2}, t \in[0,0.1], \xi \in \Gamma \\
u(x, 0, \xi) & =10 \sin \left(\pi x_{1}\right) \sin \left(\pi x_{2}\right) \xi_{1}, & x \in[0,1]^{2}, \xi \in \Gamma \\
u(x, t, \xi) & =0, & x \in \partial[0,1]^{2}, t \in[0,0.1], \xi \in \Gamma
\end{aligned}
$$

where $a(x, \xi)=0.5\left(\bar{a}(x)+\sum_{j=1}^{12} \sqrt{\lambda_{j}} X_{j}(x) \xi_{j}\right)$ the first 12 terms of the K-L expansion with $\xi_{j} \sim U[-1,1]$ and $X_{j}(x), \lambda_{j}$ given by the covariance function

$$
\operatorname{Cov}(x, y)=\exp \left(-\|x-y\|^{2}\right) / l^{2}, \quad l=0.5
$$

- adaptivity parameters: Leja knots - nested, $m\left(i_{n}\right)=2 i_{n}-1$ - adding 2 points in every adaptivity step - function $\mathrm{f}$ :

$$
f(\xi)=-\sum_{j=1}^{12} \sqrt{\lambda_{j}}\left\langle X_{j} \nabla \bar{u}_{R}, \nabla U\right\rangle_{L^{2}(D)} \xi_{j}-\sum_{i=1}^{R} \sum_{j=1}^{12} \sqrt{\lambda_{j}}\left\langle X_{j} \nabla U_{i}, \nabla U\right\rangle_{L^{2}(D)} \xi_{j} Y_{i}(\xi),
$$

where $U=\left(U_{1}, \ldots, U_{R}\right)^{T}, Y=\left(Y_{1}, \ldots, Y_{R}\right)^{T}$.

\begin{tabular}{c|c|c|c} 
type of grid & no. of collocation points & max no. of pts in each dim & error indicator \\
\hline adapted sparse grid & 3465 & {$[7,5,5,5,3,3,3,5,3,3,5,5]$} & $7.88 \mathrm{e}-2$ \\
tensor grid & 23625 & {$[7,5,5,5,3,3,3]$} & $4.85 \mathrm{e}-1$ \\
tensor grid & 354375 & {$[7,5,5,5,3,3,3,5,3]$} & $4.33 \mathrm{e}-1$ \\
isotropic sparse grid & 6188 & {$[6,6,6,6,6,6,6,6,6,6,6,6]$} & $3.88 \mathrm{e}-1$
\end{tabular}

Table 1: Discretization parameters: $\mathbb{P}_{1}$ FEM space $(51 \times 51$ points $)$, semi-implicit Euler scheme $\left(10^{3}\right.$ time steps), rank $R=13$, knots: Leja - nested for sparse grids, Gauss-Legendre for tensor grids. Multiindex set for isotropic sparse grid: $\sum_{n} i_{n}-1 \leq w$. Reference solution: $u_{h}\left(\cdot, 0.09, y_{l}\right), l=1, \ldots, 9$ computed with a very small time step ( $10^{5}$ steps) at 9 uniformly-randomly chosen points $y_{l}$. Error indicator: $\sum_{l}\left\|u_{R}\left(\cdot, 0.09, y_{l}\right)-u_{h}\left(\cdot, 0.09, y_{l}\right)\right\|_{L^{2}(D)} /\left\|u_{h}\left(\cdot, 0.09, y_{l}\right)\right\|_{L^{2}(D)}$ averaged over $y_{l}, l=1, \ldots, 9$

\section{References}

[1] Musharbash, E., Nobile, F., Zhou, T., Error analysis of the dynamically orthogonal approximation of time dependent random PDEs, SIAM J. SCI. COMPUT.,37(2), A776-A810, 2015 [2] Nobile, F., Tamellini, L., Tesei, F., Tempone R, An adaptive sparse grid algorithm for elliptic PDEs with lognormal diffusion coefficient, Sparse Grids and Applications - Stuttgart, 2014 [3] Musharbash, E., Dynamical Low Rank approximation of PDEs with random parameters, PhD dissertation, no. 7813, École Polytechnique Fédérale de Lausanne, 2017 